\title{
The Role Played by Internal Locus of Control and Self Regulation in Learning Outcome
}

\author{
Mme Lysinge Magdalene Limunga \\ Head of Department, Applied Science in Education G.T.T.C Limbe, Cameroon
}

The purpose of this paper is to present information gathered from some studies investigating locus of control and related constructs that will help educators as a whole and teachers in particular to work with learners, so as to foster internal locus control, self motivated and self-regulated learning behaviours. This discourse is much needed because much has changed in recent years. Gone are the days when society used to blame learners failure on school inadequacy and teacher incompetence. Learners today are expected to develop self motivated learning behaviour, develop learning strategies and take responsibility for their learning outcome.

Locus of control, as apply to school age children has evolved over time .The major theorist of this concept is Rotter J (1966). According to him, how one perceives learning outcomes and attributes responsibility for them influences one's self-concept and the motivation to succeed. Locus of control therefore, is a phenomenon of attributing outcome to either internal or external factors: meaning the place where the learner puts responsibility for performance results. Those who ascribe their learning outcome to internal factors such as personal effort put on studying, skill and competence manifest internal locus of control. Such learners' make statements like "I did not study hard enough" I need more background for this course or I was careless when reading the test question, etcetera, when they do not perform well as expected.

On the other hand, those who feel that their academic outcomes are determined by external factors, which are beyond their control, have external locus of control. Such learners make statements like "the teacher was unfair or hates me, I was not lucky on that day or the time was too short, etcetera. Locus of control is viewed as a characteristic, which develops with age. A development increase in internality, from very young learners through high school. The dependence of young children upon others for instrumental help and emotional support is a necessary condition for early development.

Crandall, Zimmermann and Paulsen (1995) asserted that it would not be surprising then to find that preschool children and infants strongly depend on adults especially caregivers and the concomitant acquisition of independent problem solving techniques that are equally important requisite of normal personality development. But with age and experience, most children begin to feel that their own actions are often instrumental in attaining the reinforcement they receive. The above authors further assessed that the beliefs of elementary and secondary school children concerning "how" and if they (learners) rather than other people, were responsible further intellectual success and failure are always bound to succeed.
EposiDoh, is a case study of many children who attribute learningdifficulties to assigned reading. She complains that it is too long and difficult, that if only the passage were short and lot easier, she would be successful. Instead of focusing internally on her need to increase effort and proficiency, she is worried and avoids reading, which makes her feel increasingly powerless. If EposiDoh were more centered on her own need for developing reading skills and less focused on external factors such as assignment difficulty, she would be more likely to seek help and improve her academic performance. Her beliefs about the cause of her performance are keeping her from making satisfactory progress. She has lost faith and control in her academic ability. Even though she was successful in other areas, she attributed it to luck. She lacks self-confidence and also attributes her success in exams on luck. So she is not putting enough effort, and does not increase study time.

From the above case study one deduces the fact that, the explanations one gives for successes and failures actually influences subsequence performances. The power of selfbelief is very strong. If people succeed, it may not be reinforcing or encouraging for future performance, if they attribute success to factors outside their control that is, external locus of control. Werner's theory of attribution postulates that, how a person explains success or failure is more important than how often the person succeeds or fails. When learners take personal responsibility for success in high. In case of failure such learners would blame themselves following inadequate effort or un-preparedness this shows internal locus of control and better of succeeding in subsequent assessments. But if he/she continuously blames the teachers and other situational occurrenciesit is indicative of the fact that he/she will do little to improve on study skill. Internal locus of control demonstrates high efficiency level in achievement.

To maintain high expectation for success, most motivation theorists believe that success should be attributed to high ability and failure to lack of effort rather than on personal adequacies. Motivation theorists suggest that since the goal of educators is to optimize learning, particular support should be given to low achieving learners and those who do not accept personal responsibility for their learning. No matter the instructional approach used in the classroom, it is essential that learner" s personal control of learning be developed. They should be provided with opportunities to exercise control and choice over carefully selected tasks, with constant and immediate feedback. It is important, to that if low achieving children are not attended to on time, they may develop what psychologists call learned helplessness. Learned - helplessness is an experience in which a person learns that his/her efforts have little to do with the outcome of a situation. Learners with such 
experience may learn that when faced with difficulty, it is useless to try to surmount them : instead ,they become helpless, passive Repressed ,and pessimistic (ZickKubin 1995). Children who suffer from habitual failure syndrome as a result of past assignments or tests always have external locus of control because, they may not work harder or persist longer when they encounter difficulties. The concept of learned helplessness was first discovered in animal research by Martin Seligman and StievenMairer (1995). They found out that when dogs were unable to escape electrical

Sortant'T to that' if low shocks, they (dogs) simply gave up trying. Even when escape was later possible they still could not increase effort at trying to escape. Learners who have internal locus of control are usually self regulatory learners. Self-regulatory processes are tools for self -improvement as they encourage the learners to direct attention, to set and adjust their goals and to guide their course of learning more effectively. Some ways that learning could be enhanced within this framework include; distinguishing between helplessness was

effective and ineffective performances and then linking it back to identify the behaviour that may have caused it, identify inadequate learning strategies, managing study time more effectively and fostering reflective thin :. For this to be successful learners must establish learning goals, access whether or not being met, and then modify their approach to learning if necessary. Educators and parents should help learners to set realistic goals. For instance if NalovahAngwafor's long term goal is to become a medical doctor, she has to stay motivated and develop a positive belief system about her own ability to succeed and be willing to adapt to her goals.

She may need to find a support network composed of peers, teachers, counselors and parents as well. For her to succeed she may also need to master her environment, control and regulate her activities. As she does this she will gradually become more confident about her ability to succeed. She may also need to analyse her long -term goal of becoming a doctor by breaking it down into smaller parts and finding a way of connecting it more directly to her current class work and assuming personal responsibility for learning outcome. Zimmerman and Paullsen (1995) outline four phases for facilitating and developing a process of self-regulated learning.

First, learners must establish a baseline of expectations for themselves in a given learning situation from which they can set goals. For instance NalovahA, could begin to monitor herself and record how long it takes to complete certain types of assignments and which types are most difficult for her. See Figure 1; serving as an example of a self-monitoring strategy.

\begin{tabular}{|l|l|l|l|l|l|l|l|}
\hline Date & Assignment & Time Started & Time Finished & Where & With Whom Obstacles & Self belief/Efficacies \\
\hline & & & & & & & \\
\hline
\end{tabular}

Second, she could be shown how to structure her monitoring activities with a protocol such as a self-questioning strategy that provides immediate feedback on her learning. She could regularly ask herself toarticulate the main theme of a class lecture or to summarise a chapter in the text -Next, she would need to become more independent and prepare protocols that are more individualized for her needs.

Finally, she may arrive at a level of self-regulated monitoring, be able to generalize the process, and transfer it to other subjects areas.

The basic assumption reflected in all self-regulating activities is that the learner has established a set of goals. Without goals there are no standards against which to evaluate ones performance. Two types of learning goals that are frequently used include mastery and performance.

Hagen and Weiristen (1995) describe mastery goals as having a primary focus related to learning the material at hand; meanwhile performance goals focus on the outcome, with learning serving only as a means to an end.

When a learner is engaged m trying to attain mastery goals, she is more apt to seek out challenges and put forth effort. Such learners tend to be more relaxed and less anxious, more collaborative and better able to use effective study methods than those who are motivated simply by grades. Grade - Oriented learners who are driven by performance goals frequently conclude that failure is due to their inadequacies and this belief may cause them to exhibit patterns of helplessness in future learning situations. This tendency may explain their lack of persistence when difficulties arise. Grade oriented learners may construct goals that are so low that they are assured of meeting them or so high that failure is almost expected. To maintain a riskfree environment, such learnerse.ga student with little background knowledge in science has little to lose when setting a goal of becoming a doctor. The goal is most likely so far out of reach that no one could reasonably expect him to reach it, and consequently it involves little risk. To successfully engage in a process of self regulated learning, learners mustbelieve that they are active partners in the learning process. They must see themselves as being in control of their learning and have access to repertoire of strategies that can be used when modifications are needed. To be successful monitor of their own learning, they also need to construct goals that provide an appropriate set of standards against which to evaluate their Progress. This behaviour is essential to effective learning, and they must be taught and integrated in all learning environments. After some research findings and theoretical foundations, the following guidelines have been put forward to enable teachers help learners to develop self - regulation in Selfregulatory learning enables learners to actually control their learning. This means learners begin with a meta-cognitive understanding of what they would have to do, in other to meet their goals of becoming proficient in an area. Here, there is a strong belief in her ability to a dream come true and because of the belief; she puts in effort that will lead her to achieve success. Her goals are personal; no one should impose goals on a learner. Personal goals are structured for intermediate success. A significant component of this is creating an environment that is optimal for learning. This includes her need to reach out for assistance and restructure her perceptions of her own role as a learner. This would be 
enhanced if teachers suggest study techniques for organizing the materials to be learned and tell learners how to read notes through a practice model. They could also review notes and highlight significant ideas together, which would provide a model for the importance of going through notes selectively. Another model would be how to read a chapter in a text. Learners need to set learning goals that enhance internal motivation. Students who have imposed goals sometimes lack internal motivation. But those with personal goals study hard because they are internally motivated to achieve high standards in their work. They are selfdetermined because they are doing something out of their will, not because of external rewards; they equally take personal responsibility for their learning outcome, including reaching the goals they have set. Harter,(1996) carrying out some studies on motivation emphasizes that, an important goal of parenting and teaching should be to help children develop greater internalization and intrinsic motivation, as the children grow older.

All of these do not mean to undermine Cihe pertinence of extrinsic motivation in learning. But this paper encourages learners to be intrinsically motivated which are based on internal factors as self-determination, challenges and efforts.

Self -regulatory learning requires a minimal level of prior knowledge in a given domain. Teachers who expect young learners to develop the skills may be unrealistic in the demands on learners, instead they should be do as much modeling as possible to help the learners become familiar with ways of thinking, and organizing ideas and facts. They should provide study aids, timings and summaries that can facilitate learners' attempts at self-regulation. It is significant at this level to remind learners of their prior knowledge and demonstrate how to make connections to new materials.

Self -regulated learning develops overtime as learners increase their sense of self-efficacy. Most often learners who lack articulated goals have a low self-efficacy; such learners have no experiences with success in formal educational settings. They lack the belief that they can meet their expectations. Helping such learners to articulate meaningful and attainable goals would be a good step. Teachers should provide opportunities for such learners to succeed and to help make connections between that success and their efforts. Once the teacher has provided some initial modeling, by asking learners to try it out in pairs or small groups with the subject matter would be an effective method. Thus practice should not only be used at the beginning to provide positive feedback and to validate its significance, but to make connection:-) to materials, build internal commitment and to encourage transference across the curriculum In other for this to happen, teachers should not only provide direct instruction, but also opportunities for learners to experience success with various self -regulated learning strategies and to make the connections with desired outlook. This is only possible when learners teach one another from their own success. Facilitating small group discussions in class that focus on the strategies that worked well and the ones that did not work following a test is good example of allowing learners themselves to make connections.

Fellow audience, (protocol duly respected) this presenter strongly believes that locus of control intrinsic motivation and self-regulated learning set premise for instruction based on " minds on, hands on" as stipulated by the new pedagogic approach (N.P.A)

Thanks for listening.

\section{REFRENCES}

[1] Rotter, J.B. (1966) Generalized Expectancies for Internal versus External Control of Reinforcement. Psychological Monographs, 80, 1-28.

[2] Zimmerman B. J., (2000), Self-Efficacy: An Essential Motive to Learn, Contemporary Educational Psychology n. 25 .

[3] Zimmerman, B. J. (2002). Becoming a self-regulated learner: An overview. Theory into Practice, 41(2), 64-70.

[4] [4] Zimmerman, B. J. \& Schunk, D. H. (1976). Selfregulated learning and academic achievement: Theory, research, and practice. New York, Springer Verlag. Zimmerman B. J. \& Schunk D. H. (Eds.) (2003). Educational psychology: A century of contributions. Mahwah, NJ, US: Erlbaum.

[5] Liking and Loving: An Invitation to Social Psychology. ResearchGate https://www.researchgate.net/.../232467354_Liking_an d_Loving_An_Invitation_to_S...

[6] Zick Rubin ... Trying to explain the difference between liking and loving, Rubin (1970 Rubin (, 1973 proposes romantic love .... ( Baumeister \& Leary, 1995;Buss, 1983;Heider, 1958;Hill, 1987;Jones, 1964), and the desire to convey competence ...

[7] Steven D. Maurer's research works | University of Oregon, Oregon (UO https://www.researchgate.net/scientificcontributions/7631420_Steven_D_Maurer

[8] Steven D. Maurer's 22 research works with 503 citations and 1615 reads, ... and promising theory from the academic HRM literature (Hom \& Griffeth 1995, pp. 